\title{
Conocimiento de cáncer de mama y factores sociodemográficos, acceso y atención en salud en mujeres consultantes en centros oncológicos, Cartagena - Colombia
}

\author{
Conhecimento do câncer de mama e fatores sociodemográficos, acesso \\ e cuidados de saúde em mulheres consultoras em centros de câncer, \\ Cartagena - Colômbia
}

Knowledge of breast cancer and sociodemographic factors, access and
health in women consultants in cancer centers, Cartagena - Colombia

Verónica del Carmen Castro Bocanegra

veronica.castro@curnvirtual.edu.co | https://orcid.org/o0oo-0002-1138-4463

Rafael de Jesús Tuesca Molina

rtuesca@uninorte.edu.co | https://orcid.org/0000-0003-3095-7199

${ }^{1}$ Corporación Universitaria Rafael Núñez, Programa de Instrumentación Quirúrgica. Cartagena, Colombia.

2 Universidad del Norte, Programa de Maestría en Salud Pública. Barranquilla, Colombia.

a Magister en Salud Pública pela Universidad del Norte.

b Doctorado en Medicina Preventiva y Salud Publica pela Universidad Autónoma de Madrid.

\section{RESUMEN}

Identificar los factores relacionados al conocimiento del estado actual de la enfermedad en mujeres con cáncer de mama y factores sociodemográficos, diagnóstico, tratamiento y atención del cáncer de mama en instituciones oncológicas, Cartagena-Colombia. Estudio descriptivo transversal; participaron 211 mujeres con cáncer de mama, mediante una encuesta estructurada. El Odd Ratio (OR) fueron calculados usando SPSS versión 23.o. Se encontró una asociación estadística entre la práctica del autoexamen de mama con el poseer estudios de educación superior y pertenecer al nivel contributivo; además, una mujer menor de 50 años con diagnostico confirmado de cáncer y que tenga un nivel educativo superior entró con una acción de tutela. Se identificaron lagunas derivadas de la estratificación social, segmentación geográfica y acceso a mecanismos de información telefónica. Por lo tanto, las condiciones de vulnerabilidad bajo nivel educativo y pobreza marcan diferencias y denotan oportunidades perdidas para la detección, atención y tratamiento.

Palabras claves: Atención; Diagnóstico; Tratamiento; Cáncer de mama; Conocimiento. 


\section{RESUMO}

Identificar fatores relacionados ao conhecimento do estado atual da doença em mulheres com câncer de mama e fatores sociodemográficos, diagnóstico, tratamento e cuidado do câncer de mama em instituições oncológicas, Cartagena-Colômbia. Estudo descritivo transversal; 211 mulheres com câncer de mama participaram através de uma pesquisa estruturada. Foi encontrada uma associação estatística entre a prática do auto-exame das mamas e o fato de ter uma educação superior e pertencer ao nível contributivo; além disso, uma mulher com menos de 50 anos de idade com diagnóstico confirmado de câncer e com um nível de educação superior entrou com uma ação de tutela. São identificadas as lacunas derivadas da estratificação social, segmentação geográfica e acesso a mecanismos de informação telefônica. Portanto, as condições de vulnerabilidade, baixo nível educacional e pobreza marcam diferenças e denotam a perda de oportunidades de detecção, cuidado e tratamento.

Palavras-chave: Cuidados; Diagnóstico; Tratamento; Cancro da mama; Conhecimento.

\section{ABSTRACT}

To identify factors related to knowledge of the current state of the disease in women with breast cancer and sociodemographic factors, diagnosis, treatment and care of breast cancer in oncological institutions, Cartagena-Colombia. Cross-sectional descriptive study; 211 women with breast cancer participated through a structured survey. A statistical association was found between the practices of breast self-examination and having a higher education and belonging to the contributory level; in addition, a woman under 50 years old with a confirmed diagnosis of cancer and with a higher education level uses guardianship action by right. Gaps derived from social stratification, geographical, health system segmentation and access to telephone information mechanisms are identified. Therefore, the conditions of vulnerability, low educational level and poverty mark differences and denote lost opportunities for detection, care and treatment.

Keywords: Attention; Diagnosis; Treatment; Breast cancer; Knowledge.

Contribuição dos autores: Ambos autores trabajaron en la planeación, ejecución y análisis de la investigación. Así como en la construcción del artículo.

Declaração de conflito de interesses: Los autores declaran no presentar conflictos de interés.

Fontes de financiamento: No se contó con financiaciones para la ejecución de la investigación.

Considerações éticas: Esta investigación fue aprobada por el Comité de Ética de la Universidad del Norte y legalizada en acta de evaluación № 138 y certificada en consenso por sus miembros integrantes Agradecimentos/Contribuições adicionais: Se agradece a las mujeres con cáncer de mama, profesionales de la salud, instituciones participantes y demás colaboradores que con su sensibilidad y aportes enriquecieron este estudio.

Histórico do artigo: submetido: 14 maio 2020 | aceito: 05 jun. 2020 | publicado: 22 mar. 2021.

Apresentação anterior: não há.

Licença CC BY-NC atribuição não comercial. Com essa licença é permitido acessar, baixar (download), copiar, imprimir, compartilhar, reutilizar e distribuir os artigos, desde que para uso não comercial e com a citação da fonte, conferindo os devidos créditos de autoria e menção à Reciis. Nesses casos, nenhuma permissão é necessária por parte dos autores ou dos editores. 


\section{INTRODUCCIÓN}

El cáncer de mama es el quinto tipo de cáncer que causa el mayor número de fallecimientos anuales a nivel mundial (571 ooo defunciones) y es considerado como el más frecuente en mujeres de países desarrollados y en vía de desarrollo (ORGANIZACIÓN MUNDIAL DE LA SALUD, 2018; 2021). En Latinoamérica, representa el $27 \%$ de los nuevos casos y el $16 \%$ de las muertes por cáncer. De igual manera, en Estados Unidos y Canadá, presenta el 24\% de los nuevos casos y el 14\% de la mortalidad en mujeres (CANCER..., 2018). En Colombia, según datos de la Organización Panamericana de la Salud (OPS), esta patología en el año 2016 estimó una tasa de 17,3 por 100000 habitantes (ORGANIZACIÓN PANAMERICANA DE LA SALUD, 2019), y para el año 2014 se reportó una tasa de 11,49 por 100000 habitantes lo que denota un incremento a nivel nacional (SISTEMA INTEGRAL DE INFORMACIÓN DE LA PROTECCIÓN SOCIAL, 2019), siendo Bolívar el segundo departamento de la Costa Atlántica con el mayor número de pacientes fallecidas por esta enfermedad (INSTITUTO NACIONAL DE CANCEROLOGÍA ESE, 2017).

Los determinantes que influyen en la aparición de la enfermedad pueden ser prevenibles y modificables, mejorando las condiciones socioeconómicas, cambiando a hábitos saludables y promocionando programas de salud adaptados y adecuados a la región donde se aplican teniendo presente el nivel cultural y social de la misma (INSTITUTO NACIONAL DE SALUD, 2019. AGUILAR et al., 2012).

La probabilidad de supervivencia de un paciente con cáncer de mama es mayor y la mortalidad se reduce si el diagnóstico y el tratamiento es precoz. Un diagnóstico temprano debe incluir un estado de conciencia de la situación de salud y acceso a la atención médica, una evaluación oportuna y estadificada; y un acceso al tratamiento. Pero un diagnóstico tardío genera deterioro de la calidad de vida de las mujeres, esto originado posiblemente por las barreras de acceso a los servicios de diagnóstico y tratamiento oportuno. Estas barreras pueden trabajar en doble sentido, convirtiéndose en replicadores o reductores de la desigualdad, dependiendo de la disponibilidad en el acceso, utilización y calidad del servicio (TAMBORERO-CAO, 2015).

En Colombia, según la Ley 100 de 1993 y sus modificaciones, no es evidente la implementación de la atención primaria en salud en todo el territorio. Oficialmente, el 93\% de la población está afiliada al sistema de salud mediante la oferta estratificada por la capacidad de pago. El régimen contributivo permite un paquete de salud a cargo de una entidad prestadora de salud privada bajo la vigilancia del Estado, ofertado para la población con capacidad de pago y a sus beneficiarios del afiliado; mientras que para el régimen subsidiado, a cargo del Estado, se brinda un paquete diferencial a personas sin capacidad de pago; y para otras entidades como son fuerzas militares, magisterio y empresa de petróleos se vinculan al régimen especial, con característica de excepcional en salud con diferentes mecanismos para prestación, oferta y financiación. No obstante, en el país, el 6,7\% no tiene afiliación y en estos hacen parte 1,7 millones de migrantes provenientes de Venezuela. Este modelo de financiación, administración y provisión de servicios de salud del país reproduce la fragmentación e inequidad del sistema de salud (AGUDELO CALDERÓN; CARDONA BOTERO; ORTEGA BOLAÑOS; ROBLEDO MARTÍNEZ, 2011). En este sentido, existe evidencia de las dificultades que presentan las mujeres del régimen subsidiado y la mayor prevalencia de realización de la mamografía en las mujeres que pertenecen al régimen contributivo y especial (PIÑEROS PETERSEN; PARDO RAMOS; GAMBOA GARAY; HERNÁNDEZ SUAREZ, 2010). Por lo anteriormente expresado, el factor socioeconómico, la posición laboral, nivel educativo, edad y etnia son factores diferenciales para recibir una atención y con calidad dentro del sistema sanitario (POBLANO-VERÁSTEGUI; FIGUEROAPEREA; LÓPEZ-CARRILLO, 2004).

En Cartagena, el Departamento Administrativo Distrital de Salud (Dadis) es el encargado de realizar el seguimiento técnico a las instituciones de salud de la ciudad, verificando que cada una de ellas cumpla con la normatividad vigente y además, ejecuten las capacitaciones respectivas a los usuarios afiliados dentro de 
los programas de promoción y prevención. Desde el Programa de Salud Sexual y Reproductiva del DADIS se desarrollan actividades para promover el autoexamen de mama y detectar oportunamente signos y síntomas relacionados con la neoplasia mamaria. De igual forma, existen redes de apoyo en la ciudad tales como fundaciones, comunidades religiosas y demás organizaciones sin ánimo de lucro que brindan una ayuda a las mujeres con cáncer de mama para abordar esta problemática desde el aspecto emocional y social y para contribuir en la mejoría de su calidad de vida. Pero a pesar de estos esfuerzos, la mortalidad por esta patología ocupa la tasa más alta, posicionándose en primer lugar en estos últimos 7 años de manera consecutiva (15.6 muertes por cada 100.000 mujeres) (CABALLERO; FERNANDEZ; BRITT, 2018).

El presente estudio tiene por objetivo identificar los factores asociados con el conocimiento del estado actual de la enfermedad oncológica en mujeres con cáncer de mama, relacionadas con factores sociodemográficos que influyen en el diagnóstico, tratamiento y atención del cáncer de mama en instituciones públicas y privadas de salud en la ciudad en Cartagena.

\section{MATERIAL Y MÉTODOS}

Se trata de un estudio descriptivo transversal, en una población universo de 463 pacientes que padecen de cáncer de mama atendidas en dos centros oncológicos de la ciudad de Cartagena; de la cuales se seleccionaron 211 pacientes de diferentes regímenes de salud (subsidiado, contributivo, prepagadas y régimen especial), correspondientes al 95\% del cálculo de la muestra, escogidas de forma aleatoria mediante una técnica de muestreo aleatorio simple. Se tuvo como criterios de inclusión a mujeres en edad mayor o igual a 28 años con diagnóstico confirmado de cáncer de mama, residentes en la ciudad de Cartagena y que aceptaron la participación en el estudio de manera voluntaria.

Se aplicó una encuesta estructurada (autodiligenciada) a las participantes durante la asistencia a la consulta médica con el especialista; este consta de 25 preguntas enfocadas en determinantes estructurales (variables independientes) de tipo individual: edad, estado civil; socioeconómico: estrato, procedencia, nivel educativo, ocupación laboral, tipo de afiliación al sistema general de seguridad social en salud (SGSSS); conducta personal: práctica habitual del autoexamen de mama, asistencia a consultas médica para valoración de las mamas; acceso a los servicios de salud: recursos financieros y distancia entre el lugar de vivienda y el punto de atención en salud; la percepción del sistema de salud (variables dependientes), tales como conocimiento de la enfermedad: estadio del cáncer al inicio del tratamiento, estadio actual en el que se encuentra el cáncer; responsabilidad del equipo de salud: médico general, médico especialista, subespecialista; calidad de la atención recibida y oportunidad de la atención: tutelas impuestas para acceder a la atención, motivos de la demora en el proceso de diagnóstico y tratamiento. Este instrumento fue validado mediante técnica de validación por expertos.

Se creó una base de datos en Excel 2010 para la recopilación de la información. Se realizó un análisis descriptivo e inferencial con el paquete informático SPSS versión 20.0 en español, para la presentación y análisis de la misma; la cual se realizó mediante tablas uni y bivariadas, de acuerdo con los objetivos específicos.

En las categorías de análisis se compararon ser menor o mayor a 50 años de edad, si conviven con pareja, si pertenecen al estrato social medio/alto o bajo, si tienen estudios superiores o básicos, si son procedentes de área urbana o rural, si trabajan fuera del hogar o en él y si están afiliadas al régimen contributivo o subsidiado.

Las tablas univariadas se presentan con la medida de frecuencia y porcentaje. Las tablas bivariadas presentan la asociación estadística por medio del OR, Chi Cuadrado y Grados de libertad, Intervalos de confianza al 95\% y se el valor significativamente estadístico fue de $\mathrm{p}<0.05$. 
La convocatoria de participación se realizó a través de oficios formales a las instituciones que participaron en el estudio y las pacientes firmaron un consentimiento informado.

\section{RESULTADOS}

De acuerdo a las características sociodemográficas, el 35,5\% ( $\mathrm{n}=75)$ de la población en estudio pertenece al grupo de edad de 51 a 60 años. El 62,1\% (n=131) vive con su pareja ya sea casado o en unión libre. El 64\% ( $n=135)$ se auto identificó en el estrato bajo. El nivel educativo predominante oscila entre la primaria y la secundaria, y representa el 54,9\% del porcentaje acumulado, $28,4 \%(n=60)$ y $26,5 \%(n=56)$ respectivamente. El 78,7\% ( $\mathrm{n}=166)$ refiere residir en la zona urbana, más de la mitad $(58,3 \%)$ se dedica a las actividades del hogar y el 61,6\% ( $n=130)$ pertenece al régimen contributivo incluyendo en este grupo los afiliados a medicina prepagada y los de régimen especial (Ver Tabla 01).

Con respecto al nivel de conocimiento de su enfermedad, el 78,7\% ( $\mathrm{n}=166)$ de las mujeres encuestadas manifestaron que conocían la etapa de la enfermedad en la cual se encontraban al momento del diagnóstico y un 90,5\% (n=191) la conoce en la actualidad. El 64,5\% (n=136) afirman que se realizaban el autoexamen de mamas de forma regular antes de ser diagnosticadas y el 54,5\% ( $n=115)$ se hacia la valoración por un médico general o especialista. Con respecto a los factores de atención en salud, en el grupo de estudio indican que para ellas la atención médica ha sido buena $(86,7 \% \mathrm{n}=183)$ y una baja proporción de mujeres ha tenido que recurrir a un medio judicial para la resolución de derechos en salud (18,5\% $n=39)$. Al valorar los antecedentes de familiares directos con cáncer de mama, el 64,0\% ( $\mathrm{n}=135)$ no presentó antecedentes. El 50,7\% ( $\mathrm{n}=107)$ de las encuestadas indica que le generó un impacto psicológico el acontecimiento de padecer cáncer y el 92,0\% (n=194) no pensó en algún momento de su vida padecer de cáncer de mama.

Al indagar en el grupo de mujeres que conocen la etapa de la enfermedad una vez que fueron diagnósticas, de las 211 encuestadas, sólo 166 mujeres (78,6\%) manifestaron conocerla. De estas, el 37,3\% ( $\mathrm{n}=62)$ cuando las diagnosticaron estaban en la etapa temprana de la enfermedad, seguido en un 30,7\% $(n=51)$ diagnosticas in situ. De la muestra total, 191 mujeres $(90,5 \%)$ identificaron conocer la etapa de la enfermedad en la que se encuentra actualmente; de ellas, el $62,3 \%(n=119)$ manifiestan estar en un periodo de recuperación, pasando por un estado postmastectomía, reconstrucción mamaria ó postcuadrantectomía.

Relacionado al concepto de la disponibilidad de recursos financieros que posee la paciente para acceder a los servicios de salud, el 44,1\% ( $\mathrm{n}=93$ ) cuenta con los recursos para cancelación de copago, mientras que el 26,1\% ( $n=55)$ no cuenta con esos recursos. Para el 54\% ( $n=114)$ su lugar de residencia queda lejos del punto de atención en salud donde recibe la misma, por lo cual requiere emplear medio de transporte para llegar al centro de salud $(89,1 \% \mathrm{n}=188)$. El más empleado es el transporte público (71,1\% n=150). El ingreso mensual predominante corresponde a un salario mínimo $(40,8 \% \mathrm{n}=86)$.

Referente a la oportunidad en la atención, el 51,7\% ( $n=109)$ corresponde a mujeres que indican que en su centro de salud no dispone de programas de promoción y prevención del cáncer de mama, lo que muestra un desconocimiento de la existencia y función de estos programas. En una menor proporción de estas mujeres $(25,1 \% \mathrm{n}=53)$ indican que su centro de salud o EPS se comunicó con ellas después del resultado del diagnóstico de cáncer de mama y sólo el 14,7\% $(\mathrm{n}=31)$ manifiesta que su centro de salud o EPS se comunicó con ellas al incumplir una cita. El 97,6\% (n=206) corresponde a mujeres que realizaron todos los exámenes diagnósticos y de laboratorio que el médico o especialista les formuló.

$\mathrm{Al}$ asociar las variables dependientes con los factores sociodemográficos de la población objeto de estudio, se encontraron 6 asociaciones estadísticamente significativas: 
1. Las mujeres menores de 50 años conocen el doble de su enfermedad al momento del diagnóstico comparado con las mujeres mayores de 50 años (OR:2,62; IC al 95\%: 1,21 - 5,63; p-valor: o,012). De igual forma, las que poseen un nivel de estudio superior la conocen dos veces más que las que tienen un estudio básico (OR:2,36; IC al 95\%: 1,12 - 4,99; p-valor: 0,021) y desempeñarse en labores fuera del hogar, les permite conocer su enfermedad tres veces más en relación con las mujeres que permanecen en el hogar. (OR:2,97; IC al 95\%: 1,30 - 6,79; p-valor: 0,007). (Ver Tabla 02).

2. Las mujeres de origen urbano conocen el estadio actual de su enfermedad dos veces más que las de origen rural (OR:2,77; IC al 95\%: 1,05 - 7,27; p-valor: 0,032). (Ver tabla 02).

3. En relación con la práctica del autoexamen de mama antes del diagnóstico, se estima que las mujeres con estudios de educación superior (OR:2,37; IC al 95\%: 1,28 - 4,38; p-valor: 0,005), y de nivel contributivo (OR:1,86; IC al 95\%: 1,04 - 3,32; p-valor: 0,033), practicaban el doble la autoexploración en comparación con las mujeres de estudios básicos y de régimen subsidiado. (Ver Tabla 02).

4. En este estudio, la variable edad, específicamente el grupo de menores de 50 años, tiene una gran relevancia. Existe el doble de posibilidad que una mujer menor de 50 años con diagnostico confirmado de cáncer (OR:2,48; IC al 95\%: 1,22 - 5,03; p-valor: 0,010) y con un nivel educativo superior (OR:2,68; IC al 95\%: 1,31 - 5,46; p-valor: o,005), solicite una acción de tutela u otra medida judicial para hacer valer sus derechos en salud y recibir un tratamiento oncológico oportuno en comparación con las mujeres mayores de 50 años y con estudios de básica primaria y bachillerato. (Ver Tabla 02).

5. Los centros de atención incluyendo las EPS, se comunican dos veces más con las mujeres mayores de 50 años que con las mujeres menores de 50 años posterior al diagnóstico (OR:2,32; IC al 95\%: 1,154 - 4,68; p-valor: 0,016), y se comunican el doble de veces con las mujeres que viven en el área urbana que con las residentes en el área rural (OR:2,56; IC al 95\%: 1,02 - 6,46; p-valor: 0,04). (Ver Tabla 03).

6. Las pacientes que residen cerca del centro de atención cuando pertenecen al estrato medio/alto (OR:2,12; IC al 95\%: 1,20 - 3,76; p-valor: 0,009), con un nivel educativo superior (OR:1,87; IC al 95\%: 1,07 - 3,28; p-valor: 0,027) y al régimen contributivo (OR:1,96; IC al 95\%: 1,11 - 3,47; p-valor: $\mathrm{o}, 019)$ tienen el doble de la atención, en comparación con las mujeres que reciben atención oncológica de bajo estrato, nivel básico en educación y afiliación subsidiada. (Ver Tabla 04).

No se encontró asociación entre los factores sociodemográficos y la valoración de las mamas por un médico antes del diagnóstico, tener antecedentes directos con cáncer, haber sufrido un impacto psicológico generado por padecer cáncer de mama, pensar en algún momento de su vida que le daría cáncer de mama, conocer la disposición de programas de promoción y prevención en su EPS, comunicación del centro de salud con la paciente al incumplir una cita médica y la realización de todos los exámenes diagnósticos y de laboratorios. No existió una asociación significativa con la variable independiente del estado civil con respecto a las variables dependientes estudiadas. 
Tabla 01 - Características sociodemográficas de la población femenina diagnosticada con cáncer de mama. Cartagena-Colombia

\begin{tabular}{|c|c|c|c|}
\hline $\begin{array}{l}\text { Variable } \\
\text { sociodemográfica }\end{array}$ & Categorías & $\begin{array}{l}\text { Frecuencia } \\
*(n=211)\end{array}$ & $\begin{array}{l}\text { Porcentaje } \\
\quad(\%)\end{array}$ \\
\hline \multirow{6}{*}{ Edad (años) } & $>81$ & 2 & 0,9 \\
\hline & $30-40$ & 32 & 15,3 \\
\hline & $41-50$ & 49 & 23,2 \\
\hline & $51-60$ & 75 & 35,5 \\
\hline & $61-70$ & 42 & 19,9 \\
\hline & $71-80$ & 11 & 5,2 \\
\hline \multirow{3}{*}{ Estrato socioeconomico } & Alto & 12 & 5,7 \\
\hline & Bajo & 135 & 64 \\
\hline & Medio & 64 & 30,3 \\
\hline \multirow{5}{*}{ Nivel educativo } & Ninguno & 12 & 5,7 \\
\hline & Primario & 60 & 28,5 \\
\hline & Secundaria & 56 & 26,5 \\
\hline & $\begin{array}{l}\text { Vocacional - } \\
\text { Técnica }\end{array}$ & 38 & 18 \\
\hline & Profesional & 45 & 21,3 \\
\hline \multirow{2}{*}{ Procedencia } & Rural & 45 & 21,3 \\
\hline & Urbana & 166 & 78,7 \\
\hline \multirow{5}{*}{ Ocupación } & $\begin{array}{c}\text { Empleada } \\
\text { Formal No Prof }\end{array}$ & 4 & 1,9 \\
\hline & $\begin{array}{l}\text { Empleada } \\
\text { Formal Prof }\end{array}$ & 40 & 19 \\
\hline & $\begin{array}{l}\text { Empleada No } \\
\text { Formal }\end{array}$ & 29 & 13,7 \\
\hline & Hogar & 123 & 58,3 \\
\hline & Pensionada & 15 & 7,1 \\
\hline \multirow{2}{*}{ Estado civil } & $\begin{array}{l}\text { No Tiene } \\
\text { Pareja }\end{array}$ & 80 & 37,9 \\
\hline & $\begin{array}{l}\text { Vive Con la } \\
\text { Pareja }\end{array}$ & 131 & 62,1 \\
\hline \multirow{2}{*}{ Afiliación } & Contributivo & 130 & 61,6 \\
\hline & Subsidiado & 81 & 38,4 \\
\hline
\end{tabular}

Fuente: Los autores (2020).

Nota: * $(n=211)$. 
Tabla 02 - Asociación entre variables sociodemográficas con aspectos relacionados con conocimiento de diagnóstico, enfermedad, autoexamen de mama, valoración de las mamas y empleo de acción judicial. Cartagena-Colombia

\begin{tabular}{|c|c|c|c|c|c|c|c|c|c|c|c|c|c|c|c|}
\hline \multirow[t]{2}{*}{$\begin{array}{l}\text { Variables } \\
\text { sociodemo } \\
\text { graficas }\end{array}$} & \multicolumn{3}{|c|}{$\begin{array}{l}\text { Conocimiento de la etapa } \\
\text { de la enfermedad al } \\
\text { momento del diagnóstico }\end{array}$} & \multicolumn{3}{|c|}{$\begin{array}{l}\text { Conocimiento de la etapa } \\
\text { actual de la enfermedad }\end{array}$} & \multicolumn{3}{|c|}{$\begin{array}{c}\text { Práctica del autoexamen } \\
\text { de mama antes del } \\
\text { diagnóstico }\end{array}$} & \multicolumn{3}{|c|}{$\begin{array}{l}\text { Valoración de las mamas } \\
\text { por un médico }\end{array}$} & \multicolumn{3}{|c|}{$\begin{array}{l}\text { Empleo de medios } \\
\text { judiciales }\end{array}$} \\
\hline & OR & I.C & p- Valor & OR & I.C & p-Valor & OR & I.C & p-Valor & OR & I.C & p- Valor & OR & I.C & p- Valor \\
\hline $\begin{array}{l}\text { Grupos de } \\
\text { edad }<50 \text { a }\end{array}$ & 2,62 & $\begin{array}{c}1,21- \\
5,63\end{array}$ & 0,012* & 0,85 & $\begin{array}{c}0,32- \\
2,23\end{array}$ & 0,743 & 0,93 & $\begin{array}{c}0,52- \\
1,66\end{array}$ & 0,815 & 1,28 & $\begin{array}{l}0,73- \\
2,25\end{array}$ & 0,371 & 2,48 & $\begin{array}{l}1,22 \\
-5,03\end{array}$ & $0,01 *$ \\
\hline Estado civil & 1,11 & $\begin{array}{l}0,57- \\
2,19\end{array}$ & 0,745 & 0,51 & $\begin{array}{c}0,18 \\
1,47\end{array}$ & 0,237 & 1,05 & $\begin{array}{c}0,58- \\
1,87\end{array}$ & 0,867 & 0,96 & $\begin{array}{c}0,55- \\
1,69\end{array}$ & 0,91 & 0,85 & $\begin{array}{c}0,41- \\
1,73\end{array}$ & 0,657 \\
\hline $\begin{array}{l}\text { Estrato } \\
\text { social }\end{array}$ & 1,5 & $\begin{array}{l}0,73- \\
3,08\end{array}$ & 0,261 & 1,77 & $\begin{array}{c}0,61- \\
5,09\end{array}$ & 0,335 & 1,2 & $\begin{array}{c}0,66- \\
2,17\end{array}$ & 0,546 & 0,96 & $\begin{array}{c}0,54- \\
1,69\end{array}$ & 0,903 & 1,91 & $\begin{array}{c}0,94- \\
3,87\end{array}$ & 0,067 \\
\hline $\begin{array}{l}\text { Nivel } \\
\text { educativo }\end{array}$ & 2,36 & $\begin{array}{c}1,12- \\
4,99\end{array}$ & $0,021 *$ & 2,82 & $\begin{array}{l}0,91- \\
8,75\end{array}$ & 0,091 & 2,37 & $\begin{array}{c}1,28- \\
4,38\end{array}$ & $0,005^{*}$ & 1,24 & $\begin{array}{c}0,71- \\
2,17\end{array}$ & 0,434 & 2,68 & $\begin{array}{c}1,31- \\
5,46\end{array}$ & $0,005^{*}$ \\
\hline Procedencia & 1,96 & $\begin{array}{c}0,93- \\
4,13\end{array}$ & 0,071 & 2,77 & $\begin{array}{l}1,05- \\
7,27\end{array}$ & $0,032 *$ & 0,88 & $\begin{array}{c}0,44- \\
1,77\end{array}$ & 0,727 & 1,87 & $\begin{array}{c}0,96- \\
3,65\end{array}$ & 0,062 & 2,06 & $\begin{array}{c}0,75- \\
5,61\end{array}$ & 0,195 \\
\hline Ocupación & 2,97 & $\begin{array}{c}1,30- \\
6,79\end{array}$ & $0,007 *$ & 1,66 & $\begin{array}{l}0,57- \\
4,76\end{array}$ & 0,461 & 1,59 & $\begin{array}{c}0,86- \\
2,93\end{array}$ & 0,135 & 1,1 & $\begin{array}{c}0,62- \\
1,96\end{array}$ & 0,724 & 1,82 & $\begin{array}{c}0,90- \\
3,69\end{array}$ & 0,093 \\
\hline Afiliación & 1,73 & $\begin{array}{c}0,89- \\
3,37\end{array}$ & 0,102 & 2,11 & $\begin{array}{c}0,83- \\
5,34\end{array}$ & 0,108 & 1,86 & $\begin{array}{c}1,04- \\
3,32\end{array}$ & $0,033^{*}$ & 1,39 & $\begin{array}{c}0,80- \\
2,44\end{array}$ & 0,238 & 1,74 & $\begin{array}{c}0,81- \\
3,73\end{array}$ & 0,148 \\
\hline
\end{tabular}

Fuente: Los autores (2020).

Notas: OR: Odds ratio.

I.C: Intervalo de confianza.

P. valor. ${ }^{*} \mathrm{p}<0,05$. 
Tabla o3 - Asociación entre variables sociodemográficas y variables antecedentes, impacto acceso y comunicación en mujeres con cáncer de mama atendidas en centros oncológicos de Cartagena-Colombia

\begin{tabular}{|c|c|c|c|c|c|c|c|c|c|c|c|c|c|c|c|}
\hline \multirow[t]{2}{*}{$\begin{array}{l}\text { Variables } \\
\text { Sociodemograficas }\end{array}$} & \multicolumn{3}{|c|}{$\begin{array}{l}\text { Antecedentes familiares } \\
\text { con cáncer }\end{array}$} & \multicolumn{3}{|c|}{$\begin{array}{c}\text { Impacto psicológico } \\
\text { generado }\end{array}$} & \multicolumn{3}{|c|}{$\begin{array}{l}\text { Pensar en algún } \\
\text { momento que tendría } \\
\text { cáncer de mama }\end{array}$} & \multicolumn{3}{|c|}{$\begin{array}{l}\text { Disposición de } \\
\text { programas de } \\
\text { promoción y } \\
\text { prevención }\end{array}$} & \multicolumn{3}{|c|}{$\begin{array}{l}\text { Comunicación del } \\
\text { centro de salud con la } \\
\text { paciente posterior al } \\
\text { diagnóstico }\end{array}$} \\
\hline & OR & I.C & p- Valor & OR & I.C & p- Valor & OR & I.C & p- Valor & OR & I.C & p-Valor & OR & I.C & p- Valor \\
\hline Grupos de Edad & 0,71 & $\begin{array}{c}0,40- \\
1,27\end{array}$ & 0,259 & 0,73 & $\begin{array}{c}0,41- \\
1,27\end{array}$ & 0,267 & 0,88 & $\begin{array}{c}0,32 \\
- \\
2,41\end{array}$ & 0,805 & 0,79 & $\begin{array}{l}0,45 \\
- \\
1,38\end{array}$ & 0,421 & 2,32 & $\begin{array}{c}1,15- \\
4,68\end{array}$ & $0,016^{*}$ \\
\hline Estado Civil & 1,28 & $\begin{array}{c}0,71- \\
2,30\end{array}$ & 0,405 & 0,96 & $\begin{array}{c}0,55- \\
1,68\end{array}$ & 0,903 & 0,86 & $\begin{array}{c}0,31 \\
- \\
2,36\end{array}$ & 0,773 & 1,34 & $\begin{array}{c}0,77 \\
- \\
2,35\end{array}$ & 0,297 & 1,01 & $\begin{array}{c}0,53- \\
1,91\end{array}$ & 0,975 \\
\hline Estrato Social & 1,5 & $\begin{array}{c}0,84- \\
2,68\end{array}$ & 0,167 & 1,44 & $\begin{array}{l}0,82- \\
2,54\end{array}$ & 0,201 & 1,26 & $\begin{array}{c}0,46 \\
- \\
3,48\end{array}$ & 0,644 & 1,2 & $\begin{array}{c}0,68 \\
- \\
2,11\end{array}$ & 0,517 & 0,88 & $\begin{array}{c}0,46- \\
1,70\end{array}$ & 0,719 \\
\hline Nivel Educativo & 1,42 & $\begin{array}{c}0,80- \\
2,51\end{array}$ & 0,228 & 0,99 & $\begin{array}{c}0,57- \\
1,72\end{array}$ & 0,98 & 0,82 & $\begin{array}{c}0,29 \\
- \\
2,33\end{array}$ & 0,722 & 1,36 & $\begin{array}{c}0,78 \\
- \\
2,37\end{array}$ & 0,274 & 1,38 & $\begin{array}{c}0,74- \\
2,60\end{array}$ & 0,306 \\
\hline Procedencia & 1,02 & $\begin{array}{l}0,51- \\
2,04\end{array}$ & 0,942 & 1,73 & $\begin{array}{c}0,88- \\
3,38\end{array}$ & 0,105 & 0,62 & $\begin{array}{c}0,20 \\
- \\
1,87\end{array}$ & 0,396 & 1,94 & $\begin{array}{c}0,98 \\
- \\
3,85\end{array}$ & 0,053 & 2,56 & $\begin{array}{l}1,02- \\
6,46\end{array}$ & $0,04 *$ \\
\hline Ocupación & 1,66 & $\begin{array}{c}0,92- \\
2,99\end{array}$ & 0,085 & 1,39 & $\begin{array}{c}0,79- \\
2,47\end{array}$ & 0,249 & 1,76 & $\begin{array}{c}0,65 \\
- \\
4,78\end{array}$ & 0,26 & 1,25 & $\begin{array}{c}0,71 \\
- \\
2,21\end{array}$ & 0,432 & 0,96 & $\begin{array}{c}0,50- \\
1,85\end{array}$ & 0,911 \\
\hline Afiliación & 1,73 & $\begin{array}{l}0,95- \\
3,15\end{array}$ & 0,069 & 1,38 & $\begin{array}{c}0,79- \\
2,42\end{array}$ & 0,248 & 0,67 & $\begin{array}{c}0,25 \\
- \\
1,83\end{array}$ & 0,443 & 1,18 & $\begin{array}{c}0,68 \\
- \\
2,07\end{array}$ & 0,541 & 1,44 & $\begin{array}{c}0,74- \\
2,78\end{array}$ & 0,275 \\
\hline
\end{tabular}

Fuente: Los autores (2018).

Notas: OR: Odds ratio.

I.C: Intervalo de confianza.

P. valor. ${ }^{*} \mathrm{p}<0,05$. 
Tabla o4 - Asociación entre variables sociodemográficas y aspectos del entorno hacia la atención y manejo. Cartagena-Colombia

\begin{tabular}{|c|c|c|c|c|c|c|c|c|c|}
\hline \multirow[t]{2}{*}{$\begin{array}{l}\text { Variables } \\
\text { Sociodemograficas }\end{array}$} & \multicolumn{3}{|c|}{$\begin{array}{c}\text { Comunicación del } \\
\text { centro de salud con la } \\
\text { paciente al incumplir } \\
\text { una cita }\end{array}$} & \multicolumn{3}{|c|}{$\begin{array}{l}\text { Realización de todos los } \\
\text { exámenes diagnósticos } \\
\text { y de laboratorio }\end{array}$} & \multicolumn{3}{|c|}{$\begin{array}{l}\text { Lugar de residencia } \\
\text { cerca al centro de } \\
\text { atención }\end{array}$} \\
\hline & OR & I.C & p- Valor & OR & I.C & p- Valor & OR & I.C & p- Valor \\
\hline Grupos de Edad & 0,84 & $\begin{array}{c}0,38- \\
1,82\end{array}$ & 0,66 & 0,39 & $\begin{array}{c}0,04- \\
3,58\end{array}$ & 0,651 & 1,01 & $\begin{array}{l}0,58 \\
-1,77\end{array}$ & 0,946 \\
\hline Estado Civil & 1,59 & $\begin{array}{c}0,69- \\
3,65\end{array}$ & 0,27 & 1,09 & $\begin{array}{l}0,17- \\
6,69\end{array}$ & 1 & 0,98 & $\begin{array}{l}0,56 \\
-1,71\end{array}$ & 0,949 \\
\hline Estrato Social & 1,14 & $\begin{array}{l}0,52- \\
2,50\end{array}$ & 0,735 & 0,36 & $\begin{array}{c}0,06- \\
2,24\end{array}$ & 0,353 & 2,12 & $\begin{array}{c}1,20 \\
-3,76\end{array}$ & $0,009 *$ \\
\hline Nivel Educativo & 1,54 & $\begin{array}{l}0,71- \\
3,32\end{array}$ & 0,264 & 0,97 & $\begin{array}{l}0,15- \\
5,94\end{array}$ & 1 & 1,87 & $\begin{array}{l}1,07 \\
- \\
3,28\end{array}$ & $0,027 *$ \\
\hline Procedencia & 0,91 & $\begin{array}{c}0,36- \\
2,29\end{array}$ & 0,854 & 0,97 & $\begin{array}{c}0,94- \\
0,99\end{array}$ & 0,587 & 1,72 & $\begin{array}{c}0,87 \\
- \\
3,41\end{array}$ & 0,114 \\
\hline Ocupación & 1,04 & $\begin{array}{c}0,47- \\
2,32\end{array}$ & 0,911 & 0,78 & $\begin{array}{l}0,12- \\
4,83\end{array}$ & 1 & 0,87 & $\begin{array}{c}0,49 \\
- \\
1,55\end{array}$ & 0,651 \\
\hline Afiliación & 1,36 & $\begin{array}{l}0,60- \\
3,07\end{array}$ & 0,447 & 1,07 & $\begin{array}{l}0,17- \\
6,55\end{array}$ & 1 & 1,96 & $\begin{array}{c}1,11 \\
- \\
3,47\end{array}$ & 0,019* \\
\hline
\end{tabular}

Fuente: Los autores (2018).

Notas: OR: Odds ratio.

I.C: Intervalo de confianza.

P. valor. ${ }^{*} p<0,05$.

\section{DISCUSIÓN}

Los hallazgos encontrados en el presente estudio revelan algunos aspectos tratados en la literatura y se identifican otros elementos que evidencian la asociación entre los factores sociodemográficos y el cáncer de mama.

En este estudio, además, se encontró que las mujeres que conocen el doble de su enfermedad al momento del diagnóstico son las menores de 50 años, las que poseen un nivel de estudio superior y si su trabajo es fuera del hogar, conocen su enfermedad tres veces más en relación con las mujeres que permanecen en el hogar. Se observa un resultado similar a lo señalado por Castillo y colaboradores (2016) donde las mujeres de 30 a 49 años obtuvieron mayor porcentaje de conocimiento sobre la técnica de autoexamen de mama, argumentando que "se debe a que a medida que avanza el ciclo vital individual aumentan las regularidades en los estilos de vida” (p. 9).

Muchas mujeres consultantes son de estrato bajo y el nivel educativo básico o nulo, por lo que en ocasiones se dificulta la comunicación médico-paciente. Por ende, existe una falta de cultura de autocuidado, falta de educación, falta de conocimiento de los derechos, deberes y funcionamiento integral del sistema de salud; puesto que no dimensionan su estado de salud y no cumplen los tratamientos, además de la influencia de los factores económicos que impiden atenderse a tiempo y con calidad. Lo referido concuerda con MejíaMeiía et al. (2007) y por Torres-Mejía, Navarro-Lechuga, Tuesca-Molina y Angeles-Llerenas (2017) en los 
cuales se establece que la educación, la edad y tipo de afiliación a la seguridad social son factores principales incidentes en el acceso a servicios curativos y preventivos. Lastimosamente 12 años después, el presente estudio continúa evidenciando este determinante en salud.

De igual manera, las mujeres manifiestan que algunos colaboradores del servicio de atención al cliente no brindan la orientación referente a la utilización de los servicios de salud, en especial para los usuarios de estratos económicos y social bajo, lo que influye a que el paciente no conozca el lugar al cual debe dirigirse.

Existe una falta de comunicación o falta de efecto de las campañas publicitarias del autoexamen de mama, dándole un significado más que el meramente médico; que se convierta en una representación del autocuidado, que refleje el bienestar e interés de la mujer por mantenerse sana y de esta manera evitar el malestar físico y emocional propio y de sus familiares que se vive al presentar la enfermedad. Es necesario que la mujer tenga confianza y despliegue capacidades para realizarse la autoexploración de sus mamas. Debe desarrollar habilidades de tipo manual, emocional y cognitivas para detectar anomalías en sus glándulas mamarias que requieran la valoración de un experto (OSPINA DIAS et al., 2011).

Martínez-Montañez, Uribe-Zúñiga y Hernández-Ávila (2009) recomiendan que, para favorecer las conductas de autocuidado, las campañas de comunicación y educación deben enfocarse en los factores protectores del cáncer de mama, promoviendo la lactancia materna, consumo de alimentos ricos en fibras, ejecución de actividad física de forma regular, control del peso, ingesta de alcohol y no automedicación de terapias de reemplazo hormonal en la menopausia.

Un aspecto importante es el impacto psicológico que genera el diagnóstico de cáncer de mama. Por ello, la falta de apoyo familiar o de una red de apoyo que les brinde el soporte psicológico que en ocasiones la EPS no les ofrece, aunque la solicite. Esta situación es descrita por Vargas, Vásquez y Mogollón (2010) que consideran este aspecto incapacitante para el uso de servicios en salud, en especial en comunidades que poseen gran carga familiar y laboral, mujeres vulnerables económicamente y mujeres desplazadas sin integración en su contexto social.

Se expresa, además, que en pleno siglo XXI, las mujeres no consultan por miedo, vergüenza o descuido. Manifiestan estar ocupadas en las labores del hogar y no se toma el tiempo para una consulta médica. Otras, por temor a que el médico le confirme un diagnóstico que por la sintomatología presentada ellas sospechan que podría ser cáncer. A estas consideraciones son las que el estudio de Giraldo y Ceballos denominan "autobarreras" (2011, p. 143) las cuales impiden el diagnóstico y tratamiento oportuno de cáncer de mama. Las autoras estiman que esas explicaciones para no acceder al sistema de salud, no se pueden explicar simple y aisladamente, solo se logra si se analiza del contexto, vivencias personales y su relación con los demás (CEBALLOS-GARCÍA; GIRALDO-MORA, 2011).

Con respecto al acceso al tratamiento del cáncer, las pacientes consideran que el factor tiempo es crucial, y existen demoras en todo el proceso. Las EPS se demoran para las autorizaciones, asignar una cita, revalorar al paciente, lo que hace que la paciente se canse de tanta tramitología. Estos resultados son coincidentes con la investigación realizadas por Rodríguez Acosta en el año 2010 (RODRÍGUEZ-ACOSTA, 2010), referente a las barreras del acceso a los servicios de salud en Colombia, donde concluye que la principal barrera está relacionada con los múltiples trámites para conseguir una cita. Tal como se encuentra organizado el Sistema General de Seguridad Social en Colombia (SGSSC), se asegura la disponibilidad de la oferta del servicio de salud, ya sea una consulta médica o un examen diagnóstico, y aunque se contemplen tiempos mínimos para la atención, algunos estudios imagenológicos y demás exámenes diagnósticos complementarios, requieren de autorizaciones administrativas. Martínez, Segura, Arias y Mateus (2012) investigaron sobre los tiempos de atención de las mujeres con cáncer de mama comprendiendo el tiempo desde el diagnostico al ingreso a la Unidad, al inicio del tratamiento, duración del tratamiento y tiempo de control post- tratamiento, encontrando un retraso en la valoración de la patología por el personal especializado de tres a cuatro 
meses; superación del tiempo para el inicio tratamiento de cerca de seis meses, tiempo que puede obedecer a retrasos en las autorizaciones y/o a la asistencia irregular de las pacientes a las citas asignadas. Este proceso incrementa los tiempos de atención, ocasionando que muchas pacientes opten por una resolución judicial, apelando al derecho de la salud y la atención con una tutela o demanda (PIÑEROS PETTERSON et al. 2011).

En Cartagena hace falta una entidad que preste integralmente la atención en salud oncológica. Las pacientes deben dirigirse a muchos lugares, en algunos casos distantes de sus hogares, para realizarse un examen clínico, un examen diagnóstico, quimioterapias, cirugía y rehabilitación. Esta situación, refleja la falta de IPS de Mediana y Alta complejidad que vuelve critica la resolución de la atención en salud de la ciudad y aún más para pacientes oncológicos. Por ello, Agudelo (2013) señala que para que se logre una detección y tratamiento oportuno de la enfermedad, se debe disponer de una red integral de atención, que cuente con recursos físicos, económicos y humano en el cual se pueda realizar todas las fases de atención del cáncer incluyendo la rehabilitación; similar a lo expresado por Restrepo-Zea, Silva-Maya, Andrade-Rivas y VH-Dover (2014) en un estudio realizado en la ciudad de Medellín, Colombia, en el cual los profesionales de la medicina indican que uno de los principales determinantes para el acceso a los servicios de salud es la capacidad instalada de la institución para ambos regímenes de salud.

Limitaciones del estudio: El presente estudio es una investigación con metodología transversal lo cual no permite explicar causalidad, por lo tanto, se deben tomar con precaución para generalizar al país.

\section{CONCLUSIÓN}

En esta investigación, se identifican lagunas e inequidades, derivadas de la estratificación social, la segmentación geográfica (urbano-rural) y el acceso a mecanismos de información telefónica. Por lo tanto, las condiciones de vulnerabilidad, bajo nivel educativo y pobreza marcan la diferencia y denotan oportunidades perdidas para la detección, atención y tratamiento.

Dado que el cáncer de mama es un problema de salud pública reconocido a nivel mundial y nacional, se requiere una intervención directa del Estado para que reevalúe las estrategias que hasta el momento no han sido eficaces para la detección precoz de la patología, interviniendo en los modos, condiciones y hábitos de vida de la población, los cuales son determinantes sociales influyentes en su salud. Por ello es fundamental difundir los resultados a fin de establecer planes de mejora desde la atención primaria. De igual manera, hacer visible los hallazgos a la academia para reflexionar el rol de la acción educativa, social e investigativa frente al cáncer de mama en el territorio son importantes.

No obstante, se precisa diseñar estrategias que garanticen un servicio oportuno y con calidad a las usuarias del sistema que se encuentren en las fases avanzadas del cáncer, logrando la mejoría sustancial de la infraestructura de los centros de salud públicos y privados de la ciudad, que estén a la vanguardia con la tecnología y con talento humano capacitado y disponible. Esto favorecería en gran manera la eliminación de lagunas de desigualdad e inequidad puesto que se dirigen las intervenciones a los 'cuellos de botella' que presenta el sistema y se distinguen en la presente investigación. 
Reciis - Revista Eletrônica de Comunicação, Informação \& Inovação em Saúde, Rio de Janeiro, v. 15, n. 1, p. 122-135, jan./mar. 2021 [www.reciis.icict.fiocruz.br] e-ISSN 1981-6278

\section{REFERÊNCIAS}

AGUILAR CORDERO, M. J.; NERI SANCHEZ, M.; PADILLA LÓPEZ, C. A.; PIMENTEL RAMÍREZ, M. L.; GARCÍA RILLO, A.; SÁNCHEZ LÓPEZ, A. M. Factores de riesgo como pronóstico de padecer cáncer de mama en un estado de México. Nutrición Hospitalaria, Madrid, v. 27, n. 5, p. 1631-1636. 2012. DOI: http:// dx.doi.org/10.3305/nh.2012.27.5.5997. Disponible en: http://scielo.isciii.es/pdf/nhv27n5/38original29.pdf. Citado en: 7 abr. 2019.

AGUDELO BOTERO, Marcela. Determinantes sociodemográficos del acceso a la detección del cáncer de mama en México: una revisión de las encuestas nacionales. Salud Colectiva, Buenos Aires, v. 9, n. 1, p. 7990, ene./abr. 2013. Disponible en: https://scielosp.org/article/scol/2013.v9n1/79-90/. Citado en: 20 oct. 2019.

AGUDELO CALDERÓN, Carlos Alberto; CARDONA BOTERO Jaime; ORTEGA BOLAÑOS, Jesús; ROBLEDO MARTÍNEZ, Rocio. Sistema de salud en Colombia: 20 años de logros y problemas. Ciência \& Saúde Coletiva, Rio de Janeiro, v. 16, n. 6, p. 2817-2828, jun. 2011. DOI: https://doi.org/10.1590/S141381232011000600020. Disponible en: https://www.scielo.br/scielo.php?script=sci arttext\&pid=S141381232011000600020\&lng=es\&tlng=es. Citado en: 20 oct. 2020.

CANCER today: data visualization tools for exploring the global cancer burden in 2018. Lyon: International Agency for Research on Cancer; Genebra: World Health Organization; 2018. Disponible en: https://gco.iarc.fr/ today/home. Citado en: 20 oct. 2020.

CASTILLO, Irma et al. Conocimientos, actitudes y prácticas sobre el autoexamen de mama en mujeres del área rural de Cartagena. Revista U.D.C.A Actualidad \& Divulgación Científica, Bogotá, v. 19, n. 1, p. 5-14, ene./jun. 2016. Disponible en: http://www.scielo.org.co/scielo.php?script=sci arttext\&pid=S012342262016000100002\&lng=en\&nrm=iso\&tlng=es. Citado en: 20 oct. 2019.

CEBALLOS-GARCÍA, Grey Yuliet; GIRALDO-MORA, Clara Victoria. "Autobarreras" de las mujeres al diagnóstico y tratamiento oportuno de cáncer de mama. Aquichan, Chía, v. 11, n. 2, p. 140-157, ago. 2011. Disponible en: https://aquichan.unisabana.edu.co/index.php/aquichan/article/view/1897. Citado en: 20 oct. 2019.

INSTITUTO NACIONAL DE CANCEROLOGÍA ESE. Información de cáncer en Colombia. Bogotá: EI Instituto, c2017. Disponible en: https://www.infocancer.co/portal/\#!/filtro mortalidad/. Citado en: 20 nov. 2019.

INSTITUTO NACIONAL DE SALUD (Colombia). CÁNCER DE MAMA Y CUELLO UTERINO EN COLOMBIA, 4. 2018, Colombia. Informe [...]. [Bogotá]: Instituto Nacional de Salud, 2019. Versión 4. Disponible en: https://www.ins.gov.co/buscador-eventos/Informesdeevento/C\%C3\%81NCER\%20DE\%20MAMA\%20Y\%20 CUELLO\%20UTERINO 2018.pdf. Citado en: 20 nov. 2019.

MARTÍNEZ-MONTAÑEZ, Olga Georgina; URIBE-ZÚÑIGA, Patrícia; HERNÁNDEZ-ÁVILA, Mauricio. Políticas públicas para la detección del cáncer de mama en México. Salud Pública de México, Cuernacava, v. 51, supl 2, p. s350-s360, ene. 2009. Disponible en: http://www.scielo.org.mx/scielo.php?script=sci_arttext\&pid=S003636342009000800028\&lng=es. Citado en: 22 nov. 2019.

MARTÍNEZ, Susan; SEGURA, Angela; ARIAS, Samuel; MATEUS, Gilbert. Caracterización de los tiempos de atención y de mujeres con cáncer de mama que asistieron a un hospital de tercer nivel, 2005-2009. Revista Facultad Nacional de Salud Pública, Medellin, v.30, n. 2, p. 183-191. 2012. Disponible en: http://www.scielo. org.co/pdf/rfnsp/v30n2/v30n2a07.pdf. Citado en: 22 oct. 2019.

MEJIA-MEJIA, Aurelio; SÁNCHEZ-GANDUR, Andrés F.; TAMAYO-RAMIREZ, Juan C. Equidad en el acceso a servicios de salud en Antioquia, Colombia. Revista de Salud Pública, Bogotá, v. 9, n. 1, p. 26-38, ene./mar. 2007. Disponible en: http://www.scielo.org.co/scielo.php?script=sci arttext\&pid=S012400642007000100005\&lng=en\&nrm=iso\&tlng=es. Citado en: 19 oct. 2019.

ORGANIZACIÓN MUNDIAL DE LA SALUD (OMS). Cáncer de mama: prevención y control. Geneva: La Organización, c2021. Disponible en: https://www.who.int/topics/cancer/breastcancer/es/. Citado en: 07 abr. 2019.

ORGANIZACIÓN MUNDIAL DE LA SALUD (OMS). Cáncer: datos y cifras. Geneva: La Organización, 2018. Disponible en: https://www.who.int/es/news-room/fact-sheets/detail/cancer. Citado en: 07 abr. 2019.

ORGANIZACIÓN PANAMERICANA DE LA SALUD (OPS). Indicadores básicos 2019: tendencias de la salud en las Américas. Washington, DC: La Organización, 2019. 
OSPINA DÍAS, J. M. et al. La no realización del autoexamen de mama en Tunja, Colombia. Enfermería Global, Murcia, v. 10, n. 23, p. 30-40, jul. 2011. DOI: http://dx.doi.org/10.4321/S1695-61412011000300003. Disponible en: http://scielo.isciii.es/scielo.php?script=sci_arttext\&pid=S1695-61412011000300003. Citado en: 19 oct. 2019

PEREIRA CABALLERO, Pedrito Tomás; SAGBINI FERNANDEZ, Antonio; SEGOVIA BRITT, Enrique. et al. Análisis de la situación de salud con el modelo de Determinantes Sociales de Salud del Distrito de Cartagena de Indias. Cartagena: Ministerio de Salud de Colombia, 2018. Disponible en: https://www. dadiscartagena.gov.co/images/docs/saludpublica/vigilancia/d2018/asis_cartagena_2018.pdf. Citado en: 20 oct. 2019.

PIÑEROS PETERSEN, Marion; PARDO RAMOS, Constanza; GAMBOA GARAY, Óscar; HERNÁNDEZ SUAREZ, Gustavo. Atlas de mortalidad por cáncer en Colombia 2010. 3. ed. Bogotá: Instituto Nacional de Cancerología ESE; Instituto Geográfico Agustín Codazzi, 2010.

PIÑEROS PETERSEN, Marion; SÁNCHEZ, Ricardo; PERRY, Fernando; GARCÍA, Oscar Armando; OCAMPO, Rocío; CENDALES, Ricardo. Demoras en el diagnóstico y tratamiento de mujeres con cáncer de mama en Bogotá, Colombia. Salud Pública de México, Cuernavaca, v. 53, n. 6, p.478-485. 2011. Disponible en: http:// www.scielo.org.mx/pdf/spm/v53n6/a03v53n6.pdf. Citado en: 22 oct. 2019.

POBLANO-VERÁSTEGUI, Ofelia; FIGUEROA-PEREA, Juan Guillermo; LÓPEZ-CARRILLO, Lizbeth. Institutional factors contributing to the utilization of breast clinical examination. Salud Pública de México, Cuernavaca, v. 46, n. 4, p. 294-305, 2004. DOI: www.doi.org/10.1590/s0036-36342004000400004. Disponible en: https://scielosp.org/article/spm/2004.v46n4/294-305/es/. Citado en: 17 ago. 2019.

RESTREPO-ZEA, Jairo Humberto; SILVA-MAYA, Constanza; ANDRADE-RIVAS, Federico; VH-DOVER, Robert. Acceso a servicios de salud: análisis de barreras y estrategias en el caso de Medellín, Colombia. Revista Gerencia y Políticas de Salud, Bogotá. v. 13, n. 27, p. 242-265, 2014. DOI: http://dx.doi. org/10.11144/Javeriana.rgyps13-27.assa. Disponible en: http://www.scielo.org.co/pdf/rgps/v13n27/v13n27a15. pdf. Citado en: 19 oct. 2019.

RODRÍGUEZ-ACOSTA, Sandra. Barreras y Determinantes del Acceso a los Servicios de Salud en Colombia. Barcelona: Universidad Autónoma de Barcelona, 2010. Disponible en: https://archivo.alde.es/ encuentros.alde.es/anteriores/xiveea/trabajos/r/pdf/059.pdf. Citado en: 19 oct. 2019.

\section{SISTEMA INTEGRAL DE INFORMACIÓN DE LA PROTECCIÓN SOCIAL. Mortalidad ajustada por Colombia y todos los departamentos, Distritos y municipios del 2005 al 2014. Bogotá: Ministerio de Salud de Colombia, [2019]. Disponible en: http://rssvr2.sispro.gov.co/caracterizacionpoblacional/ (S(fjiiff5h4duvvirdtk5lq0ka))/default.aspx. Citado en: 07 abr. 2019.}

TAMBORERO-CAO, Gaspar. Equality in primary care clinics: Equality in daily living. Revista de calidad asistencial, Barcelona, v. 30, n. 3, p. 105-107, 2015. DOI: https://doi.org/10.1016/j.cali.2015.02.002. Disponible en: https://pubmed.ncbi.nlm.nih.gov/25890868/. Citado en: 19 oct. 2019.

TORRES-MEJIA, Gabriela; NAVARRO-LECHUGA, Edgar; TUESCA-MOLINA, Rafael; ANGELES-LLERENAS, Angélica. The Epidemiological Challenges of Breast Cancer among Premenopausal Women in Limited Resource Settings. Revista de Investigación Clínica, Ciudad de México, v. 69, n.2, p. 59-65, 2017. Disponible en: https://www.medigraphic.com/cgi-bin/new/resumenl.cgi?IDARTICULO=72433. Citado en: 20 oct. 2019

VARGAS-LORENZO, Ingrid; VASQUEZ-NAVARRETE, M. Luisa, MOGOLLÓN-PEREZ, Amparo S. Acceso a la atención en salud en Colombia. Revista de Salud Pública, Bogotá, v. 12, n. 5, p. 701-712, 2010. Disponible en: http://www.scielo.org.co/scielo.php?script=sci arttext\&pid=S0124-00642010000500001\&lng=en\&nrm=iso\&t lng=es. Citado en: 20 oct. 2019. 